\title{
Results of five years study of the epidemiological characteristics of psychotic patients participated in the program of Day hospital, in the Hellenic Center for Mental Health and Research, Branch of Heraklion, Crete
}

\author{
Eftichios Michelarakis ${ }^{1,2 *}$, Charalampos Bastas ${ }^{1}$, Maria Louloudi ${ }^{1}$, Chariklia Volikou', Amalia Gourniezaki², \\ Aikaterini Micheli ${ }^{3}$, Maria Basta ${ }^{3}$, Nikolaos Makaronas ${ }^{1}$
}

From $1^{\text {st }}$ International Congress on Neurobiology and Clinical Psychopharmacology and European Psychiatric Association Conference on Treatment Guidance

Thessaloniki, Greece. 19-22 November 2009

\section{Background}

The purpose of this study was the description of the epidemiological characteristics and the results of the therapeutic interventions in the Day Hospital(DH) of Mental Health Center(MHC) of Heraklion, Crete, and more specifically for psychotic patients, over five years.

\section{Materials and methods}

Our sample consistent of 62 psychotic patients (44 men and 18 women) who participated in the $\mathrm{DH}$ of the $\mathrm{MHC}$, in groups of 10 and for six months period, between the year 2003 and 2008. Data was analyzed with SPSS 15

\section{Results}

The majority of the participants were between the age $26-35$ years $(41 \%)$ and $36-45$ years $(33,9 \%)$ old $.71 \%$ were diagnosed with psychosis, $11,3 \%$ bipolar, while $14,5 \%$ had more than one diagnoses. Among the participants $79 \%$ came from the city of Heraklion and the rest from rural areas, $92 \%$ were non married or divorced, on the other hand $94 \%$ lived with some family members. Although, $72,6 \%$ had a high school or higher level education, $87,1 \%$ were either unemployed or disabled to work. A $56,5 \%$ of our participants had been hospitalized in closed psychiatric units and $49,4 \%$ reported a family history of mental disorder. Duration of participation in DH was $>6$ months for $53,2 \%$ of our sample and $53,2 \%$ was compliant with

${ }^{1}$ Hellenic Mental Health \& Research Center of Heraklion, Crete, Greece treatment. Moreover, compliance to treatment was negatively correlated to recurrence $(\mathrm{p}=0,036)$ and positively to the duration of participation in the $\mathrm{DH}(\mathrm{P}=0,011)$. On the other hand pharmaceutical interventions included administration of antipsychotics (82,3\% atypical, 9,7\% typical), antidepressants (56,5\%), mood stabilizers (22,8\%) and benzodiazepines(25,8\%). Medications were in tablets for $79 \%$, while $16 \%$ were on intramuscular medications. Other therapeutic interventions included occupational therapy $(66,1 \%)$, group therapy $(85,5 \%)$ and crisis intervention $(90,3 \%)$.

\section{Conclusions}

Our DH mainly applies to young and middle age psychotic patients, with severe impairment of their function improving compliance to their treatment and reducing the recurrence of the disorder.

\section{Author details}

${ }^{1}$ Hellenic Mental Health \& Research Center of Heraklion, Crete, Greece. ${ }^{2}$ Technological University of Crete-Nursing Department, Greece. ${ }^{3}$ Venizelio General Hospital of Heraklion, Greece.

\section{Published: 22 April 2010}

\section{doi:10.1186/1744-859X-9-S1-S148}

Cite this article as: Michelarakis et al:: Results of five years study of the epidemiological characteristics of psychotic patients participated in the program of Day hospital, in the Hellenic Center for Mental Health and Research, Branch of Heraklion, Crete. Annals of General Psychiatry 2010 9(Suppl 1):S148. 